\title{
Can Ontologies Support the Gamification of Scripted Collaborative Learning Sessions?
}

\author{
Geiser Chalco Challco ${ }^{1,2(\bowtie)}\left(\mathbb{D}\right.$, Ig Ibert Bittencourt ${ }^{2(\bowtie)}(\mathbb{D})$ \\ and Seiji Isotani ${ }^{1(\otimes)}(\mathbb{D}$ \\ 1 University of São Paulo, São Carlos, SP 13566-590, Brazil \\ geiser@usp.br, sisotani@icmc.usp.br \\ 2 Federal University of Alagoas, Maceió, AL 57072-900, Brazil \\ ig.ibert@ic.ufal.br
}

\begin{abstract}
In the field of Computer-Supported Collaborative Learning (CSCL), scripts orchestrate the collaborative learning (CL) process to achieve meaningful interactions among the students and so improve the learning outcomes. Nevertheless, the use of scripts may cause motivational problems over time. To deal with this issue, we propose the gamification of scripted CL sessions through an ontology that encodes knowledge from game design practices and theories of motivation and human behavior. This knowledge may be used by intelligent theory-aware systems to avoid the one-size-fits-all approach, providing support for the personalization of gamification. In this paper, we reported the results obtained in an empirical study to validate our ontology-based gamification of scripted CL sessions. Findings from this study indicate that intrinsic motivation, perceived choice, and effort/importance of students were significantly better when our ontology was used to support the gamification. The learning outcomes were significantly better in scripted CL sessions gamified through our approach, with positive correlations to the intrinsic motivation and perceived choice. Based on these results, we can state that the use of ontologies provides adequate support to carry out well-thought-out gamification of scripted sessions.
\end{abstract}

Keywords: Gamification · Ontologies $\cdot$ Scripted collaboration

\section{Introduction}

In CL scenarios, the use of CSCL scripts promotes fruitful and significant interactions among students [18,21]. Despite these benefits, motivational problems may occur when a scripted CL session has a high degree of coercion/imposition. For example, when there is an over-scripting [17], the CL sessions limit the students' behaviors and actions, causing a lack of motivation because the students may feel forced to follow an unwilling sequence of interactions. To deal with this motivational problem, and others (such as the lack of interest in the contentdomain, and the learners' preference to work individually), Gamification "as the 
use of game design elements in non-game contexts" [16] has been pointed out as a novelty approach to engaging students in educational contexts [4]. However, gamification is too context-dependent $[19,29]$, so that its benefits depend on how well the game elements are linked with the pedagogical objectives of CL sessions. Gamifying CL sessions is a non-trivial task, and when the game elements are not tailored using the one-size-fits-all approach, as was indicated in other contexts, may cause detrimental to students' motivation [2], cheating [27], embarrassment [28], and lack of credibility [13].

The main difficulty of gamification, in particular for instructional designers who are novices in this approach, is that it needs knowledge from the game design practices and the theories of motivation and human behavior. Without a common representation of this knowledge in a manner that can be understood for computers, we can not build intelligent systems that support the interpretation of theories and practices related to gamification. These intelligent theory-aware systems are also responsible to guide the instructional designers in the personalization of gamification, where the theories and practices of gamification may be used to predict the effects of a gamification design in the students' motivation and engagement, and with this information, these systems can suggest the gameelements and their design that best fit for each student in a scripted CL session. Thus, employing a top-down ontology engineering approach and the model of roles proposed in [26], we developed an ontology named OntoGaCLeS ${ }^{1}$ (detailed in $[8,10])$ in which we defined structures to encode theories and practices that support the well-thought-out gamification of scripted CL sessions.

Before spending effort in the development of an intelligent theory-aware system that uses our ontology, we decided to validate the impact of using it in comparison with the approach of one-size-fits-all gamification - a gamification design in which the same game-elements are applied for all the students of CL sessions. We conducted this validation through an empirical study in which the first author mediated the interaction between the instructional designers and our ontology (simulating, thus, an intelligent system that supports the gamification process through ontologies). After to present the related work in Sect. 2, Sect. 3 delineates our ontology-based gamification of scripted CL sessions. Sect. 4 describes the formulation of this empirical study, Sect. 5 shows its operation process, Sect. 6 presents its findings, Sect. 7 discusses the interpretation of these findings, and Sect. 8 presents the conclusion and future works.

\section{Related Work}

The importance of gamification and its personalization based on game design practices and theories of human motivation and behavior has been demonstrated in different empirical studies [3, 5, 15, 24, 25,30]. However, few empirical studies were conducted to evaluate the impact of gamification in scripted CL sessions. We previously conducted two empirical studies [6,9] to explore the benefits of our proposed ontology-based gamification (Sect. 3). Finding in these studies showed

\footnotetext{
${ }^{1}$ Available at https://geiser.github.io/ontogacles/.
} 
that our approach significantly increases students' intrinsic motivation, interest/enjoyment and perceived choice when it is compared with non-gamified CL sessions. These studies also indicated that our approach reduces the students' pressure/tension, and that the dropping-out percentage of students per group is reduced through the gamification of CL sessions.

The gamification of CL session based on profiles of learners' motivation was evaluated through empirical studies conducted by Knutas et al. [22,23]. The results from these studies indicate that gamification could increase interactions, communication, and average grades of students who participated in CL discussions. However, these CL discussions were not mediated by any CSCL script.

\section{Ontology-Based Gamification of Scripted CL Sessions}

As our ontology was conceived to be the core of intelligent theory-aware systems, at least two steps are needed to gamify a scripted CL session. The $1^{\text {st }}$ step is to set the player roles $\&$ game elements for each student of the CL session based on motivational theories and player type models. This step is performed by selecting a gamification design that best fits the individual motivational goals and game-player preferences of all the students. In the ontology, the gamification designs are encoded as "Motivational strategy" into ontological structures to represent gamified CL sessions, so that an algorithm may be used to search the ontological structures that have the same pattern of individual motivational goals of students. These goals are represented in these structures as "I-mot goal (I)" - an ontological concept encoded from motivational theories, and in which we represented the expected changes in the students' motivation at the end of the scripted CL session. Figure 1 exemplifies the ontology-based gamification of a scripted CL session inspired by the theory "Cognitive Apprenticeship" [11], and delineated for two students $L_{A}$ and $L_{B}$. For this example, the ontological structure "Gamified Cognitive Apprenticeship for Yee Achiever/Yee Socializer" was selected to gamify this session because the individual motivational goals of the student $L_{A}$ match with the structures "I-mot goal (I)" shown in the frame (a) of Fig. 1. These structures represent the satisfaction of competence need and the internalization of motivation - both structures encoded from the SDT theory [14] that states that feeling challenged and being effective to do something cause the experience of control, making a person to be intrinsically motivated. Thus, as exemplified in Fig. 1, an adequate gamification design for the student $L_{A}$ with the need of competence is encoded as the motivational strategy "Gamifying by CMPT/CMPR" (Gamifying a scripted CL session by providing an environment with competition and comparison with others).

After the selection of the gamification design, the necessary and desired conditions to play player roles should be verified employing the game-player preferences of the students, and if all the students can play the roles defined by the gamification design, these roles are assigned to them. These conditions are encoded as "Player role" in the ontology, and they were encoded based on information extracted from player type models. Finally, the game elements for the 


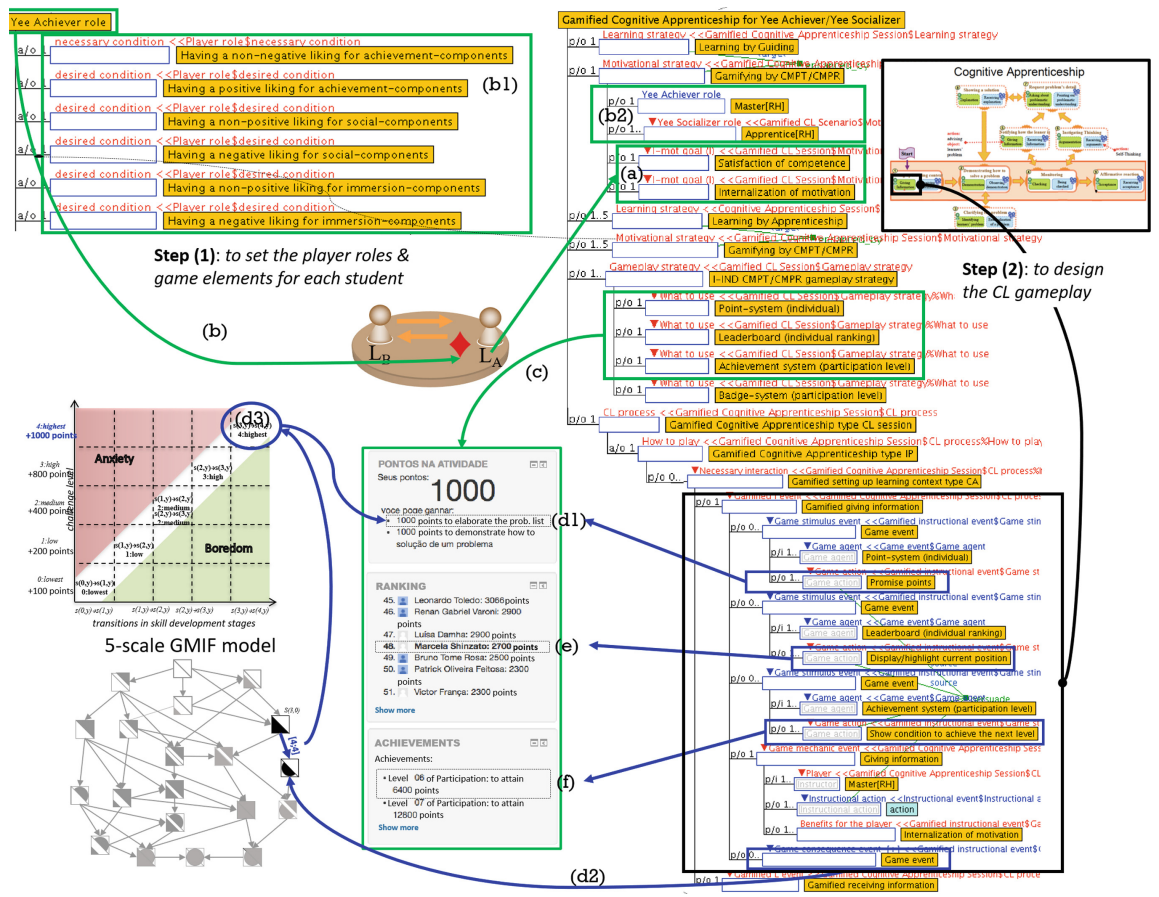

Fig. 1. Example of the ontology-based gamification of a scripted CL session

students come from the ontological structure "Gameplay strategy" - a game design practice that defines the way in which the students should interact with game elements to accomplish the individual motivational goals. The frame (b1) of Fig. 1 exemplifies the verification of necessary and desired conditions to play the player role "Yee Achiever" for the student $L_{A}$ who has the liking for achievementcomponents. Assuming that the student $L_{B}$ can also play the Yee Achiever role, no one restriction is violated in the Motivational strategy, as shown in the frame (b2) of Fig. 1. Thus, the Yee Achiever role is assigned to the student $L_{A}$, and the game elements selected for him/her are point-systems (individual), leaderboard (individual ranking), and achievement system (participation level) - elements indicated by the structure "I-gameplay" shown in the frame (c) of Fig. 1.

The $2^{\text {nd }}$ step in intelligent theory-aware system that uses our ontology is to design the CL gameplay for the CL process based on persuasive game design practices. These practices are encoded as ontological structures "gamified I_L events" that describe how the game elements should persuade the students to perform the interactions defined by a CSCL script. Thereby, an intelligent system may use this information to setting up the actions of game elements in the environment where the CL session is executed. This process is exemplified in Fig. 1 in which we present the configuration of game elements selected for the student $L_{A}$ during the instructional event "Giving information." According to 
the information encoded in the ontology, to persuade a student to give information, the game element "Point-system (individual)" should perform the game action "Promise points" as shown in the frame (d1), the game element "Leaderboard (individual ranking)" should perform the game action "Display/highlight the current position" as shown in the frame (e), and the game "Achievement system (participation level)" should perform the game action "Show condition to achieve the next level" as shown in the frame (f).

During the second step, we also need to establish a proper balance between ability and challenge. This game design practice comes from the flow theory [12], and to support the application in the gamification of scripted CL session, we developed an algorithm to build n-scale GIMF models [7]. Giving n-levels of challenges, a GIMF model distributes these levels in all the possible transitions of knowledge/skills defined by instructional/learning theories. Figure 1 exemplifies the use of a 5-scale GMIF model in a scripted CL session inspired by the Cognitive Apprentice theory. In the gamification design "Gamifying by CMPT/CMPR," the points to be obtained by a student to perform an instructional and learning actions should be directly related to the challenge levels, so that if a higher challenge is overcome, then a higher amount of points will be earned by the student. The frame (d3) of Fig. 1 shows that the number of points to be promised and given for the student during the transition $s(3, y) \rightarrow s(4, y)$ should be +1000 points based on a 5 -scale GIMF model. We defined five challenge levels because this value is the maximum number of interactions defined as gamified $I_{-} L$ events in this scripted CL session, and the max value of points was 1000 points.

\section{Formulation of the Empirical Study}

As the empirical study was formulated to validate the impact of our ontologybased gamification (detailed in Sect.3) in comparison with the one-size-fits-all gamification, we compared the students' motivation and learning outcomes in scripted CL sessions that have been gamified using these two approaches. The scripted CL sessions gamified with our approach will refer hereinafter as ontgamified CL session, whereas the scripted CL sessions gamified with the onesize-fits-all approach will refer as one-size-fits-all gamified CL sessions. Thereby, we formulated the following research questions: (1) Is the students' motivation in ont-gamified CL sessions better than in one-size-fits-all gamified CL sessions?; (2) Is the learning outcome in ont-gamified CL sessions better than in one-sizefits-all gamified CL sessions?; and (3) Are the students' motivation and learning outcomes linked on ont-gamified CL sessions?

Hypothesis Formulation: To answer the research question (1), we tested the null hypothesis, there is no significant difference of the students' intrinsic motivation in ont-gamified and one-size-fits-all gamified CL sessions, against the alternative hypothesis, $H_{1}$ :The students' intrinsic motivation is greater in ontgamified CL sessions than in one-size-fits-all gamified CL sessions. To answer 
the research question (2), we tested the null hypothesis, There is no significant difference of the students' skill/knowledge gain in ont-gamified CL sessions or non-gamified CL sessions, against the alternative hypothesis, $\mathrm{H}_{2}$ : The students' skill/knowledge gain is greater in ont-gamified CL sessions than in one-size-fits-all gamified CL sessions. To answer the research question (3), we tested the null hypothesis, There is no significant correlation between the students' intrinsic motivation and the skill/knowledge gain in ont-gamified and onesize-fits-all gamified CL sessions, against the alternative hypothesis, $H_{3}$ : There are significant correlation between the students' intrinsic motivation and the skill/knowledge gain in ont-gamified and one-size-fits-all gamified CL sessions.

Experiment Design: The empirical study was designed as a controlled experiment conducted in a real situation, in a CL activity of the course of Introduction to Computer Science, with the domain-content of Recursion, and using a CSCL script inspired by the Cognitive Apprentice theory to orchestrate the CL process. Based on this script, the students will play the CL roles of Master and Apprentice, so that this study has a $2 \times 2$ factorial design, with a randomized assignment for the types of CL session, and with a theory-driven assignment for the CL roles employing the pseudo-algorithm proposed in [20].

\section{Experiment Operation}

The empirical study was conducted in three phases (pre-test, intervention, and post-test) with 59 Brazilian undergraduate computer engineering students enrolled in the course of Introduction to Computer Science at the University of São Paulo. These students were part of a homogeneous population in the age range of 17-25 years old, sharing the same religion, social-economy status, and culture. During the conduction of this empirical study, the aspects under study and hypotheses were not informed to the students, but they were aware that the researcher would use their data with anonymity. All the materials and questionnaires employed in this study were prepared in advance, and they are available at https://bit.ly/35CpZ88. As part of the course, the students were instructed on how to participate in CL sessions orchestrated by CSCL scripts using the Scripting-forum module ${ }^{2}$, and they also answered a web-based questionnaire of the QPJ-BR instrument [1]. During this training, the students were also put in contact with the game-elements to avoid the novelty effect.

During the pre-test phase (1 week), the students' initial skill/knowledge was gathered from one programming problem task ( $\mathrm{P} 4$ - Calculate fibonacci polynomials) solved by the students using the VPL module ${ }^{3}$ in the Moodle platform, and from one multiple-choice knowledge questionnaire (p3a) answered by the students during $2 \mathrm{~h}$ at the classroom as formative evaluation.

During the intervention phase ( 4 weeks \& 3 days), the students were formed into 21 groups of 2 or 3 members with 21 masters and 38 apprentices

\footnotetext{
${ }^{2}$ Available at https://github.com/geiser/moodle_scripting_forum.

${ }^{3}$ Available at https://moodle.org/plugins/mod_vpl.
} 
assigned according to the theory-driven group formation proposed in [20]. Thus, when the students know the topic of recursion, and they known how to use recursion in the solution of a programming problem, they played the master role. Otherwise, the student played the apprentice role. After the CL role distribution, one-half of groups were randomly chosen to participate in one-size-fits-all gamified CL sessions, and the other half was chosen to participate in ont-gamified CL sessions. Thereby, 11 groups participated in ont-gamified CL sessions, and 11 groups were involved in one-size-fits-all gamified CL sessions.

The game elements were setting-up in the ont-gamified CL sessions through the approach detailed in Sect.3. Based on the individual motivational goals of students who participated in this study, the gamification designs were defined employing two ontological structures: (a) "Gamified Cognitive Apprenticeship Scenario for Master/Yee Achiever and Apprentice/Yee Achiever," and (b) "Gamified Cognitive Apprenticeship Scenario for Master/Social Achiever and Apprentice/Social Achiever." Thus, students who had more liking for achievement-components than social-components were assigned to play the Yee Achiever role, whereas students who had positive liking for social-components and achievement-components were assigned to play the Social Achiever role.

Figure 2 shows the interfaces of scripted CL sessions that have been gamified to conduct our empirical study in the Moodle platform using our plugins ${ }^{4}$ : gamepoints, game-leaderboards, game-achievements, and game badges. In scripted CL sessions that have been gamified using the structure (a), the gamification design intended to support a gameplay of individual competition in which the students acted as Yee Achiever. For these students, we provided leaderboards that display individual rankings (a4), point-systems that accumulate rewards for each individual (a3), and the win state that was defined through an achievement-system (a1) and badges of participation (a2). The gamification design for scripted CL sessions that have been gamified using the structure (2) supported a gameplay experience of individual and cooperative competition when the students played the Social Achiever role, so that we provided for them leaderboards that displayed individual rankings (b5) and collaborative rankings (b6), point-systems (b4) that accumulateed rewards for the groups and individuals, and the win state that was defined through two achievement-systems (b1) and (b2) with badges of participation and collaboration (b3).

For the one-size-fits-all gamified CL sessions, the game elements were settingup without using the ontology, so that we established the same game-elements and their design for all the students in the CL sessions, as shown in Fig. 2 (c). In these sessions, we used an individual point-system (c3), an achievement-system (c1) and badge-system (c2) for participation, and a leaderboard with individual rankings $(\mathrm{c} 4)$. The points to be given when a student perform any interaction were the same ( +500 points), and all the students received the same badge of participation at the end of the CL session. The complete setting up of gameelements and their design are detailed in https://bit.ly/3dfZdFg.

\footnotetext{
${ }^{4}$ Available at https://github.com/geiser/gamification-moodle-plugins.
} 


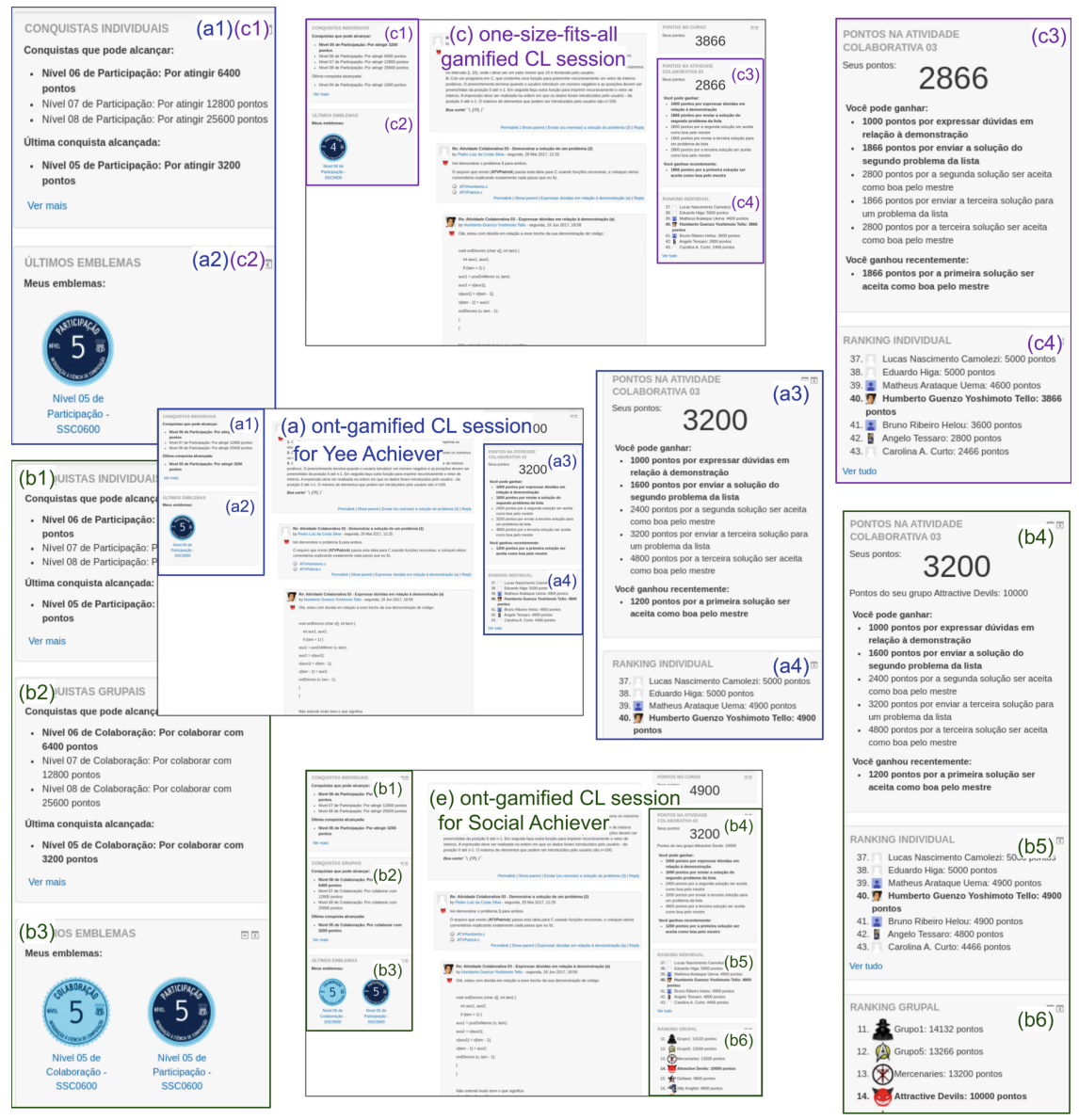

Fig. 2. Interfaces of scripted CL sessions delineated in the empirical study

During the post-test phase ( 1 week), to gather data related to the skill/knowledge, a multiple-choice knowledge questionnaire of recursion (p3c) has been answered by the students during $2 \mathrm{~h}$ at the classroom as part of the formative evaluations in the course, and three programming problem tasks (PF Programming Problem: Generation of planning poker sequence), (PG - Programming Problem: Counting palindromes) and (PH - Programming Problem: Maze solving algorithm) have been solved by the students in the Moodle platform using the VPL module. To gather data of motivation, the students answered a Web-based adapted Portuguese versions of IMI questionnaire.

\section{Findings of the Empirical Study}

Employing the responses gathered through the IMI questionnaire, we run twoway ANOVA tests and Tukey post-hoc comparisons to find significant differences 
in the dependent variables of motivation. Before this analysis, we validated the IMI questionnaire to ensure the psycho-metrically sound of its items through a CFA analysis and a reliability test obtaining a Cronbach's $\alpha=0.85$ (good) - this validation process is detailed at https://bit.ly/2xFsAS8. The students' skill/knowledge gains were estimated through the difference of scores obtained in the post-test and pre-test. With these gains, we run two-way ANOVA tests and Tukey post-hoc comparisons to find significant differences. All the results from these statistical analyses are available at https://geiser.github.io/phd-thesisevaluation/study03.

Motivation: Results from the ANOVA tests indicated significant differences on the intrinsic motivation $(F(1,47)=6.15, p=0.017)$, perceived choice $(F(1,47)=$ $8.27, p=0.006)$, and effort/importance $(F(1,47)=7.51, p=0.009)$. The tukey post-hoc comparisons show that the students' intrinsic motivation in ontgamified CL sessions (lsmean $=4.56, S E=0.149)$ was significantly greater than in one-size-fits-all gamified CL sessions $(l s m e a n=4.10, S E=0.149)$ with a p-adj. value of $p=0.023$ and Hedges' $g=0.63$ medium effect size. The perceived choice in ont-gamified CL sessions (lsmean $=4.80, S E=0.295)$ was significantly greater than in one-size-fits-all gamified CL sessions $(l s m e a n=3.60, S E=0.295)$ with $p=0.009$ and $g=0.75$ medium effect size. The effort/importance in ont-gamified CL sessions (lsmean $=5.32, S E=0.246)$ was significantly greater than in onesize-fits-all gamified CL sessions (lsmean $=4.37, S E=0.245$ ) with $p=0.007$ and $g=0.79$ medium effect size.

Learning Outcomes: According to the ANOVA tests, the effect on the students' skill/knowledge gain yielded $F(1,45)=10.77$ with $p=0.002$ indicating a significant difference between one-size-fits-all gamification and ontologybased gamification of scripted CL sessions. The Tukey post-hoc comparisons indicate that the students' skill/knowledge gain in ont-gamified CL sessions (lsmean $=1.38, S E=0.578)$ was greater than in one-size-fits-all gamified CL sessions (lsmean $=-1.26, S E=0.564$ ) with $p=0.002$ and $g=0.88$ medium effect size. For Apprentice students, their skill/knowledge gain in ont-gamified CL sessions (lsmean $=2.50, S E=0.638)$ was also greater than in one-size-fits-all gamified CL sessions (lsmean $=0.07, S E=0.585)$ with $p=0.036$ and $g=0.892$ large effect size.

Correlation of Students' Motivation and Learning Outcomes: Spearman's rank-order correlation tests were run to find significant correlations between the students' motivation and learning outcomes. According to these tests, in ont-gamified CL sessions, the skill/knowledge gain of master students was significantly strong correlated to the intrinsic motivation $(\rho=0.73$, $p=0.05)$, perceived choice $(\rho=0.85, p=0.023)$, and pressure/tension $(\rho=-0.77$, $p=0.039)$.

\section{Interpretation and Discussion of Findings}

The null hypothesis related to motivation is rejected, so that this study is evidence to support the alternative hypothesis, $H_{1}$, 'the students' intrinsic moti- 
vation is greater in ont-gamified CL sessions than in one-size-fits-all gamified CL sessions" in which the students' perceived choice and effort/importance in ont-gamified CL sessions were also greater than in one-size-fits-all gamified CL sessions. This fact is consequence of a well-though-out gamification design that, through the gamification design provided by our ontology, aligns the pedagogical objectives with the students' intrinsic motivation. As was detailed in Sect. 3, the selection of the gamification design is based on the individual motivational goals of students. These effects may also consequence of the personalization of game-elements based on information from player models and the persuasive game design applied to these game-elements. As the game-elements were set up based on a persuasive design, they easily convince the student to follow the sequence of interactions defined by the CSCL scripts during the CL process. The perceived choice and effort/importance of students in ont-gamified were greater in ontgamified CL sessions than in one-size-fits-all gamified CL sessions. A possible explanation for this fact is that students in our ont-gamified CL sessions put more effort in their actions and behaviors to be properly rewarded by the game elements because the GMIF model provides an adequate balance between the current students' skill/knowledge and perceived challenge, a balance that was not established in one-size-fits-all gamified CL sessions.

Our empirical study also constitutes evidence to support the alternative hypothesis, $H_{2}$, "the students' skill/knowledge gain is greater in ont-gamified CL sessions than in one-size-fits-all gamified CL sessions." This finding indicates that, through our gamification approach, the pedagogical benefits of scripted CL sessions are better achieved by the students. Having better pedagogical benefits in our scripted CL session is likely a consequence of increasing the students' intrinsic motivation and their autonomy sense through our ontology-based gamification approach. The evidence that supports this fact is the significant correlations found in the students' intrinsic motivation and perceived choice with the skill/knowledge gains in ont-gamified CL sessions.

\section{Conclusion and Future Works}

The findings in the empirical study reported in this paper indicate that our ontology-based approach to gamify scripted CL sessions is likely to be an efficient method to deal with motivational problems with the potential to improve the learning outcomes. In scripted CL sessions that have been gamified using our ontology-based approach, students reported to be more intrinsic motivated and with better perceived choice than in scripted CL sessions that have been gamified employing the one-size-fits-all approach. Our approach also demonstrated that raising intrinsic motivation and perceived choice in scripted CL sessions through gamification helps the students to accomplish in better learning outcomes.

Our empirical study was limited to undergraduate students (ages 17-25), to the content-domain of Recursion, and using only one CSCL script to conduct the CL sessions. As the gamification is too-context dependent, we can not generalize our findings, so additional empirical studies will be carried to validate 
the efficiency of our ontology-based approach. These further study should be conducted using other content-domains with different difficulty levels and from different courses, with other participants, and using other CSCL scripts.

\section{References}

1. Andrade, F., Marques, L., Bittencourt, I.I., Isotani, S.: QPJ-BR: Questionário para Identificação de Perfis de Jogadores para o Português-Brasileiro. In: XXVII Brazilian Symposium on Computers in Education, vol. 27, pp. 637-646. Maceio (2016)

2. Andrade, F.R.H., Mizoguchi, R., Isotani, S.: The bright and dark sides of gamification. In: Micarelli, A., Stamper, J., Panourgia, K. (eds.) ITS 2016. LNCS, vol. 9684, pp. 176-186. Springer, Cham (2016). https://doi.org/10.1007/978-3-31939583-8_17

3. Böckle, M., Novak, J., Bick, M.: Towards adaptive gamification: a synthesis of current developments. Research Papers (2017)

4. Borges, S.S., Durelli, V.H.S., Reis, H.M., Isotani, S.: A systematic mapping on gamification applied to education. In: Proceedings of the 29th Annual ACM Symposium on Applied Computing. SAC 2014, pp. 216-222. ACM (2014). https://doi. org $/ 10.1145 / 2554850.2554956$

5. Busch, M., et al.: Personalization in serious and persuasive games and gamified interactions. In: Proceedings of the 2015 Annual Symposium on Computer-Human Interaction in Play, CHI PLAY 2015, pp. 811-816. ACM (2015). https://doi.org/ 10.1145/2793107.2810260

6. Challco, G.C., Isotani, S., Bittencourt, I.I.: The effects of ontology-based gamification in scripted collaborative learning. In: 2019 IEEE 19th International Conference on Advanced Learning Technologies (ICALT), vol. 2161-377X, pp. 140-144 (2019). https://doi.org/10.1109/ICALT.2019.00043

7. Challco, G.C., Andrade, F.R.H., Borges, S.S., Bittencourt, I.I., Isotani, S.: Toward a unified modeling of learner's growth process and flow theory. Educ. Technol. Soc. 19(2), 215-227 (2016)

8. Challco, G.C., Mizoguchi, R., Bittencourt, I.I., Isotani, S.: Gamification of collaborative learning scenarios: structuring persuasive strategies using game elements and ontologies. In: Koch, F., Koster, A., Primo, T. (eds.) SOCIALEDU 2015. CCIS, vol. 606, pp. 12-28. Springer, Cham (2016). https://doi.org/10.1007/978-3-31939672-9_2

9. Challco, G.C., Mizoguchi, R., Isotani, S.: Using ontology and gamification to improve students' participation and motivation in CSCL. In: Cristea, A.I., Bittencourt, I.I., Lima, F. (eds.) HEFA 2017. CCIS, vol. 832, pp. 174-191. Springer, Cham (2018). https://doi.org/10.1007/978-3-319-97934-2_11

10. Challco, G.C., Moreira, D.A., Bittencourt, I.I., Mizoguchi, R., Isotani, S.: Personalization of gamification in collaborative learning contexts using ontologies. IEEE Latin Am. Trans. 13(6), 1995-2002 (2015). https://doi.org/10.1109/TLA. 2015.7164227

11. Collins, A.: Cognitive apprenticeship and instructional technology. In: Educational Values and Cognitive Instruction: Implications for Reform, pp. 121-138 (1991)

12. Csikszentmihalyi, M.: Flow: The Psychology of Optimal Experience. 1st edn. Harper Perennial Modern Classics, New York, July 2008 
13. Davis, K., Singh, S.: Digital badges in afterschool learning: documenting the perspectives and experiences of students and educators. Comput. Educ. 88, 72-83 (2015). https://doi.org/10.1016/j.compedu.2015.04.011

14. Deci, E.L., Ryan, R.M.: Self-Determination. The Corsini Encyclopedia of Psychology. Wiley, Hoboken (2010)

15. Deterding, S.: Eudaimonic design, or: six invitations to rethink gamification. SSRN Scholarly Paper ID 2466374, Social Science Research Network, Rochester, NY (2014)

16. Deterding, S., Dixon, D., Khaled, R., Nacke, L.: From game design elements to gamefulness: defining gamification. In: Proceedings of the 15th International Academic MindTrek Conference: Envisioning Future Media Environments, pp. 9-15. ACM (2011)

17. Dillenbourg, P.: Over-scripting CSCL: the risks of blending collaborative learning with instructional design. In: Three Worlds of CSCL. Can We Support CSCL? pp. 61-91. Open Universiteit, Nederland (2002)

18. Fischer, F., Kollar, I., Stegmann, K., Wecker, C., Zottmann, J.: Collaboration scripts in computer-supported collaborative learning. In: The International Handbook of Collaborative Learning, pp. 403-419 (2013)

19. Hamari, J., Koivisto, J., Sarsa, H.: Does Gamification Work?-A Literature Review of Empirical Studies on Gamification. In: 47th International Conference on System Sciences, HICSS 2014, pp. 3025-3034. IEEE Computer Society, Hawaii (2014). https://doi.org/10.1109/HICSS.2014.377

20. Isotani, S., Mizoguchi, R.: Theory-driven group formation through ontologies. In: Woolf, B.P., Aïmeur, E., Nkambou, R., Lajoie, S. (eds.) ITS 2008. LNCS, vol. 5091, pp. 646-655. Springer, Heidelberg (2008). https://doi.org/10.1007/978-3540-69132-7_67

21. Isotani, S., et al.: A semantic web-based authoring tool to facilitate the planning of collaborative learning scenarios compliant with learning theories. Comput. Educ. 63, 267-284 (2013). https://doi.org/10.1016/j.compedu.2012.12.009

22. Knutas, A., Ikonen, J., Maggiorini, D., Ripamonti, L., Porras, J.: Creating software engineering student interaction profiles for discovering gamification approaches to improve collaboration. In: Proceedings of the 15th International Conference on Computer Systems and Technologies, CompSysTech 2014, pp. 378-385. ACM, New York (2014). https://doi.org/10.1145/2659532.2659612

23. Knutas, A., Ikonen, J., Nikula, U., Porras, J.: Increasing collaborative communications in a programming course with gamification: a case study. In: Proceedings of the 15th International Conference on Computer Systems and Technologies, CompSysTech 2014, pp. 370-377. ACM, New York (2014). https://doi.org/ $10.1145 / 2659532.2659620$

24. Knutas, A., van Roy, R., Hynninen, T., Granato, M., Kasurinen, J., Ikonen, J.: A process for designing algorithm-based personalized gamification. Multimedia Tools Appl. 78(10), 13593-13612 (2018). https://doi.org/10.1007/s11042-018-6913-5

25. Koivisto, J., Hamari, J.: The rise of motivational information systems: a review of gamification research. Int. J. Inf. Manag. 45, 191-210 (2018). https://doi.org/10. 1016/j.ijinfomgt.2018.10.013

26. Mizoguchi, R., Sunagawa, E., Kozaki, K., Kitamura, Y.: The model of roles within an ontology development tool: Hozo. Appl. Ontol. Roles Interdisc. Perspect. 2(2), 159-179 (2007) 
27. Nunes, T.M., Bittencourt, I.I., Isotani, S., Jaques, P.A.: Discouraging gaming the system through interventions of an animated pedagogical agent. In: Verbert, K., Sharples, M., Klobučar, T. (eds.) EC-TEL 2016. LNCS, vol. 9891, pp. 139-151. Springer, Cham (2016). https://doi.org/10.1007/978-3-319-45153-4_11

28. Ohno, A., Yamasaki, T., Tokiwa, K.I.: A discussion on introducing half-anonymity and gamification to improve students' motivation and engagement in classroom lectures. In: 2013 IEEE Region 10 Humanitarian Technology Conference, pp. 215220, August 2013. https://doi.org/10.1109/R10-HTC.2013.6669044

29. Richards, C., Thompson, C.W., Graham, N.: Beyond designing for motivation: the importance of context in gamification. In: Proceedings of the First ACM SIGCHI Annual Symposium on Computer-Human Interaction in Play, CHI PLAY 2014, pp. 217-226. ACM, Canada (2014). https://doi.org/10.1145/2658537.2658683

30. Seaborn, K., Fels, D.I.: Gamification in theory and action: a survey. Int. J. Hum Comput Stud. 74, 14-31 (2015) 